\title{
Finite element modelling of helmeted head impact under frontal loading
}

\author{
PRAVEEN KUMAR PINNOJI ${ }^{1}$ and PUNEET MAHAJAN ${ }^{1}$ \\ ${ }^{1}$ Transportation Research and Injury Prevention Programme, Indian Institite of \\ Technology, Hauz Khas, New Delhi 110016 \\ e-mail:mahajan@am.iitd.ac.in
}

\begin{abstract}
Finite element models of the head and helmet were used to study contact forces during frontal impact of the head with a rigid surface. The finite element model of the head consists of skin, skull, cerebro-spinal fluid (CSF), brain, tentorium and falx. The finite element model of the helmet consists of shell and foam liner. The foam is taken as elasto-plastic, the brain is assumed to be viscoelastic and all other components are taken as elastic. The contact forces and coup pressures with helmet on the head are much lower than in the absence of the helmet. A parametric study was performed to investigate the effect of liner thickness and density on the contact forces, pressures and energy absorption during impact. For $4 \mathrm{~ms}^{-1}$ velocity, expanded poly styrene (EPS) foam of density $24 \mathrm{~kg} \mathrm{~m}^{-3}$ gave the lowest contact forces and for the velocities considered, thickness of the foam did not affect the contact forces.
\end{abstract}

Keywords. Helmeted head; finite element models; head injury; head impact.

\section{Introduction}

The human head is very vulnerable to injury, often with severe consequences. It is particularly susceptible to acceleration/deceleration and rotational forces because it is freely mobile in three dimensions and occupies a relatively unstable position, being secured only by the neck muscles and ligaments. One of the effective countermeasures to prevent head injuries in bicycle and motorcycle crashes is the use of a protective helmet. The beneficial effects of helmets in direct impact are well documented and helmets have been found to decrease the risk of head and brain injury by 70 to $88 \%$ and facial injury to the upper and mid-face by $65 \%$ (Becker 1998, Mohan \& Kothiyal 1984, Bowman et al 1982, Huston \& Sears 1981). Standards requiring helmets to conform to certain attributes and capabilities were set up as early as the 1950s and in most countries the drop test was the most common for determining impact performance of helmets. In this test, the helmet with the head form is dropped on an anvil and accelerations experienced by the head are measured. The drop test may vary from country to country in respect of size of head form, shape of anvil and the height from which it is dropped.

Numerical simulations have been used to understand the mechanism of injury in the head during impact and how the use of helmets attenuates injury. The early numerical studies 
(Bowman et al 1982, Huston \& Sears 1981) to assess the effectiveness of motorcycle helmets modelled the human body by concentrated masses joined by linkages and having energy absorbing joints. These studies investigated a wide variety of impact conditions and established that both head injury and severity of neck response were reduced by the use of helmet.

Yettram et al (1994) used Dyna3D ${ }^{\mathrm{TM}}$, a finite element (FE) tool, to simulate the impact conditions during a drop test and studied the influence of shell stiffness and liner density on mechanical behaviour of motorcycle helmet. The constitutive law for foam was arrived at on the basis of experiments at various strain rates and included the effects of foam compressibility. The authors recommended a low stiffness shell and liner of low density so that the limited space available in the helmet for deformation is used to the maximum. FE has also been used by Brands et al (1996) to study the motorcycle helmet mounted on the head form. The results were reported in the form of head injury criterion (HIC) values and accelerations of the head form. The headforms used in these studies were either made of wood or were rigid.

FE is also commonly used to model the head (Horgan \& Gilchrist 2003; Ruan et al 1994). Computed Tomography (CT) scanning and Magnetic Resonance Imaging (MRI) have been used to generate geometrical data in digital format for the head models. Once the geometry is available the material modelling of brain tissue is done next. Generally, the models do not contain all the details of the head and are much simpler than the actual head. One such 3D finite element model of the human head developed by Remy et al (2000) having skin, skull, CSF and brain is used here. The material properties of the various parts of the head were assumed to be homogeneous, isotropic and linearly elastic, except for the brain, which was assumed to be viscoelastic in nature. Remy et al simulated dynamic impact analysis of the head using the model and compared their results with the experimental results of head impacts reported in literature. They predicted contact forces between the impactor and head for the frontal impact and developed a brittle material model to simulate the compact skull bone failure. Horgan \& Gilchrist (2003) and Zong et al (2006) have also constructed three-dimensional finite element models of the human head. The former used it for simulating pedestrian accidents whereas the latter authors use a SI (Structural Intensity) approach to study power flow distribution inside the head in frontal, rear and side impacts.

We combined the FE models of head and brain by Remy, with a FE model of the helmet to determine the reduction in force experienced by the head during impact due to the presence of the helmet. The coordinates of the helmet were measured on a coordinate measuring machine. A finite element simulation of frontal impact of the combined helmet model was done on LS-Dyna ${ }^{\mathrm{TM}}$ and the contact forces, the coup and contrecoup pressures developed on the surface of the head due to impact both in the presence and in the absence of helmet were determined. A parametric study was then performed to see how changing the helmet parameters such as foam liner thickness and density of foam affect the impact forces and pressures.

\section{Helmet and head constitutive modelling}

\subsection{Helmet}

A motorcycle helmet has two major parts, namely: the outer shell and the energy absorbing liner. The outer shell, the main purpose of which is to prevent penetration of the helmet by sharp objects, is made from polymers reinforced with fibres (glass, carbon or kevlar) or thermoplastics like ABS or polycarbonate. In the FE analysis the outer shell is assumed to be made of glass fibre, reinforced plastic and modelled as a homogeneous isotropic elastic material; its properties are listed in table 1. 
Table 1. Material properties for helmet shell.

\begin{tabular}{cccc}
\hline Part & Density (tonnes $\left.\mathrm{mm}^{-3}\right)$ & Young's modulus $\left(\mathrm{N} \mathrm{mm}^{-2}\right)$ & Poisson's ratio \\
\hline Shell & $2 \cdot 0 \mathrm{E}-09$ & $80 \cdot 0 \mathrm{E}+03$ & $0 \cdot 3$ \\
\hline
\end{tabular}

\subsection{Mechanics of foam}

The liner is mainly made of expanded polystyrene (EPS) although materials such as expanded polypropylene have also been suggested (Hamouda et al 2007). The liner in the helmet is the main energy absorbing component, which prevents injury to the head during impact by reducing its deceleration. Figure 1 shows the stress-strain behavior of EPS foam. The foam depicts linear elasticity at low stresses followed by a collapse plateau, truncated by a regime of densification in which the stress rises steeply. The area under the stress-strain curve gives the energy absorbed by the liner and the stress in the liner multiplied by the contact area between the head and the liner provides the force experienced by the head during impact. The longer the plateau region, the more is the energy absorbed.

The higher the density of foam, the larger will be the energy absorbed but this will be accompanied by higher force on the head. Low density foam may reduce force on the head initially but will crush very fast and bottom out unless the thickness is very large. Therefore, the optimum density and thickness of the liner are important constituents of helmet design. The liner foam is assumed to be isotropic and modelled using an associative elasto-plastic constitutive law.

Material model \#63 available in LS-Dyna ${ }^{\mathrm{TM}}$ is used for this purpose. The model transforms the stresses into the principal stress space where the yield function is defined. If the principal stresses exceed the yield stress they are scaled back to the yield surface and transformed back to the original stress space. The yield surface and its evolution are defined by the equations:

Yield surface description:

$$
f_{t}=\left|\sigma_{i}\right|-Y=0
$$

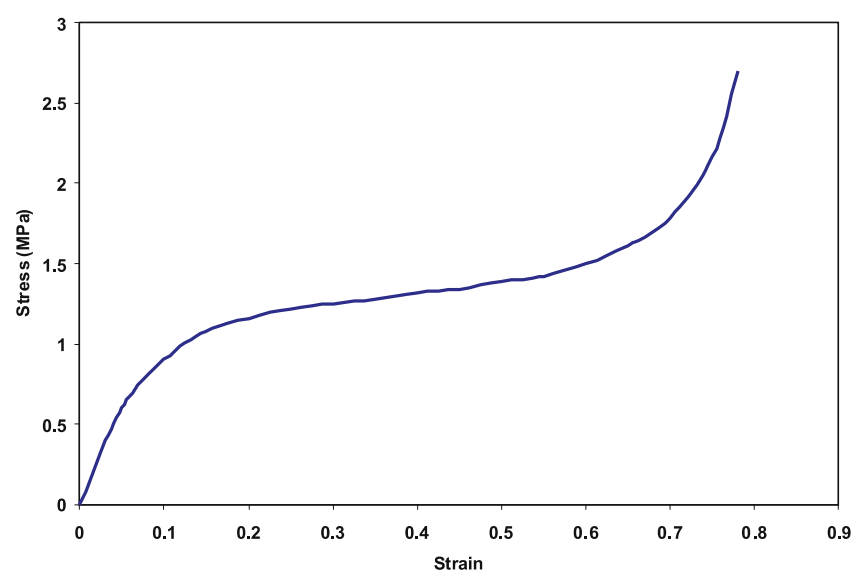

Figure 1. Stress/strain relationship for $44 \mathrm{~g}$ litre $^{-1}$ density of expanded polystyrene under quasi-static loading. 


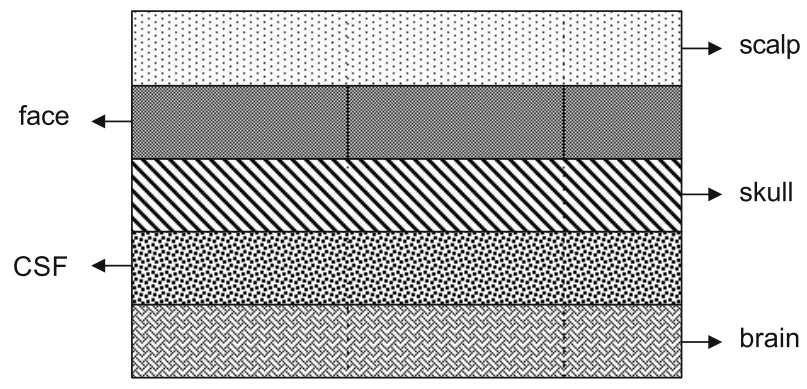

Figure 2. Schematic view of cross section of the human head, which is considered here.

Hardening formulation:

$$
\begin{aligned}
Y & =Y^{0}+H\left(e_{v}\right) \\
Y_{t} & =Y_{t}^{0}
\end{aligned}
$$

where, $Y$ is the yield stress, $Y^{0}$ is the initial compressive yield stress, $Y_{t}$ is the tensile cut off stress, $\sigma_{i}$ is the principal stress and $H$ is the strain hardening.

Here $e_{v}$ is the volumetric strain defined by natural logarithm of relative volume. An associative flow rule is assumed and the plastic strains are derived from

Flow of plastic strains:

$$
\varepsilon_{i j}^{\dot{ }}=\dot{\lambda} \frac{\partial F}{\partial \sigma_{i j}}
$$

The flow surface is the same as the yield surface.

In LS-Dyna ${ }^{\mathrm{TM}}$ the data for stress versus volumetric strain for the liner are given in tabular form and it fits the above equations to this curve. The stress-strain curves, for three different densities of liner, for uni-axial loading are taken from (Yettram et al 1994).

\subsection{Human head}

The human head is a layered structure and contains seven parts; skin, face, cranium, CSF, brain, tentorium and falx. The first five layers which are modelled here are shown in figure 2 .

The skin, the outermost layer of head, has a thickness of 5-10 mm. The skin acts as an energy absorber and load distributor in the transmission of the force to the brain. The thickness, firmness and mobility of the skin are important factors in guarding the skull from damage.

The skull (cranium) provides structural protection to the brain. It consists of separate bones which are connected by sutures but are immobile relative to one another. The bones of the skull consist of compact inner and outer tables with an intervening spongy middle layer called the 'diploe'. The thickness of bone layers vary from place to place but diploe is the thickest among these layers having low density. The skull is not a completely closed system and has a large opening called the 'foramen magnum'.

The space between the brain and the inner surface of the skull, is called the subarachnoid space (SAS) and is filled with cerebro-spinal fluid (CSF). The CSF bathes the brain and maintains a uniform pressure within the cranium in a normal head. It is a clear, colorless and nearly Newtonian liquid consisting of water, protein, gases in solution and organic constituents. On an average, $500 \mathrm{ml}$ of CSF is formed daily. It is generally accepted that the CSF 
Table 2. Material properties of head parts.

\begin{tabular}{lccc}
\hline Part & Density (tonnes mm $\left.{ }^{-3}\right)$ & Young's modulus $\left(\mathrm{N} \mathrm{mm}^{-2}\right)$ & Poisson's ratio \\
\hline Cranium & $1.80 \times 10^{-9}$ & $15 \cdot 0 \times 10^{3}$ & $0 \cdot 21$ \\
CSF & $1.04 \times 10^{-9}$ & $12 \cdot 0 \times 10^{-3}$ & $0 \cdot 49$ \\
Skin & $1.20 \times 10^{-9}$ & $16 \cdot 7$ & $0 \cdot 42$ \\
Tentorium & $1.14 \times 10^{-9}$ & 31.5 & $0 \cdot 23$ \\
Face & $3.00 \times 10^{-9}$ & $5 \cdot 0 \times 10^{3}$ & $0 \cdot 21$ \\
Falx & $1 \cdot 14 \times 10^{-9}$ & 31.5 & $0 \cdot 23$ \\
\hline
\end{tabular}

system, very likely plays an important function in protection against brain injury. Here it is treated as a soft layer with low modulus to allow the relative movement between the skull and brain and to get the reasonable impact responses. The brain is beneath the cerebro-spinal fluid in the skull. Within the brain tissue, there are a series of interconnecting cavities called the ventricular system. The Falx is a fold of dura projecting into the longitudinal fissure. The Tentorium is also a fold of dura but forming a shelf in the transverse fissures. Both these Falx and Tentorium divide the brain into left and right hemispheres and the cerebellum.

The various layers of the head are generally non-homogeneous and anisotropic. However, for modelling purposes here, they are assumed as homogeneous, isotropic and linearly elastic, except for the brain, which is assumed as viscoelastic in nature. The shear characteristics of viscoelastic behaviour of the brain are expressed by

$$
G(t)=\left(G_{\infty}+G_{0}-G_{\infty}\right) e^{-\beta t}
$$

Here $G_{\infty}$ is the long term shear modulus, $G_{0}$ is the short term shear modulus and $\beta$ is the decay factor.

The selection of biological material properties for mathematical idealizations is always a problem. The properties listed here in tables 2 and 3 are taken from the available literature. The visco-elastic properties for brain are given below.

\section{Finite element model}

The head model, used in our study contains 11939 nodes, 10396 brick elements and 2799 shell elements and has a total mass of $4.0 \mathrm{~kg}$. In the head, brain tissue, CSF and skin are modelled with 8-noded brick elements. The cranium is modelled with 4-noded shell elements. The Falx and Tentorium are modelled with 4-noded shell elements. A facial bone is modelled to obtain the realistic head inertia characteristics. The helmet model contains 29958 nodes, 21822 brick elements and has a total mass of $0.725 \mathrm{~kg}$.

Table 3. Material properties of brain.

\begin{tabular}{lccccc}
\hline Part & Density (tonnes $\left.\mathrm{mm}^{-3}\right)$ & Bulk modulus $\left(\mathrm{N} \mathrm{mm}^{-2}\right)$ & $G 0\left(\mathrm{~N} \mathrm{~mm}^{-2}\right)$ & $G I\left(\mathrm{~N} \mathrm{~mm}^{-2}\right)$ & $\beta\left(\mathrm{s}^{-1}\right)$ \\
\hline Brain & $2.0 \times 10^{-9}$ & $1.125 \times 10^{3}$ & $49.0 \times 10^{-3}$ & $16.7 \times 10^{-3}$ & 145 \\
\hline
\end{tabular}




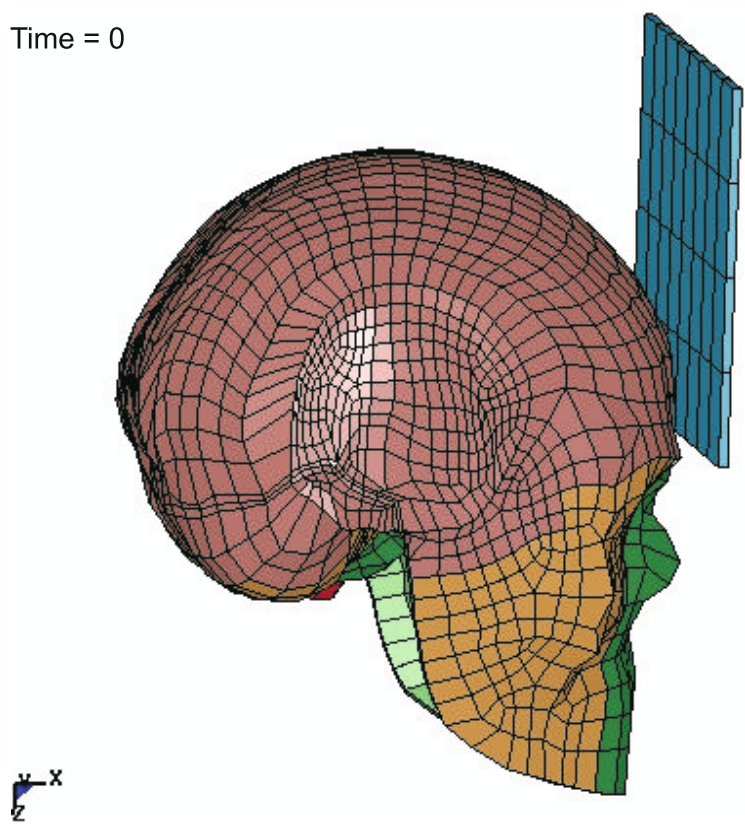

Figure 3. FE model of head impact with flat rigid surface.

Average measured values of helmets sold in India had thickness of foam liner and shell $18 \mathrm{~mm}$ and $3 \mathrm{~mm}$ respectively. Contact interactions were used between the head and helmet and between the helmet and rigid surface to prevent interpenetration of these surfaces. A velocity of $4 \mathrm{~ms}^{-1}$ was given to the helmet-head system in frontal direction. Figures 3 and 4 show FE models of the head without and with a helmet undergoing frontal impact with a flat rigid surface.

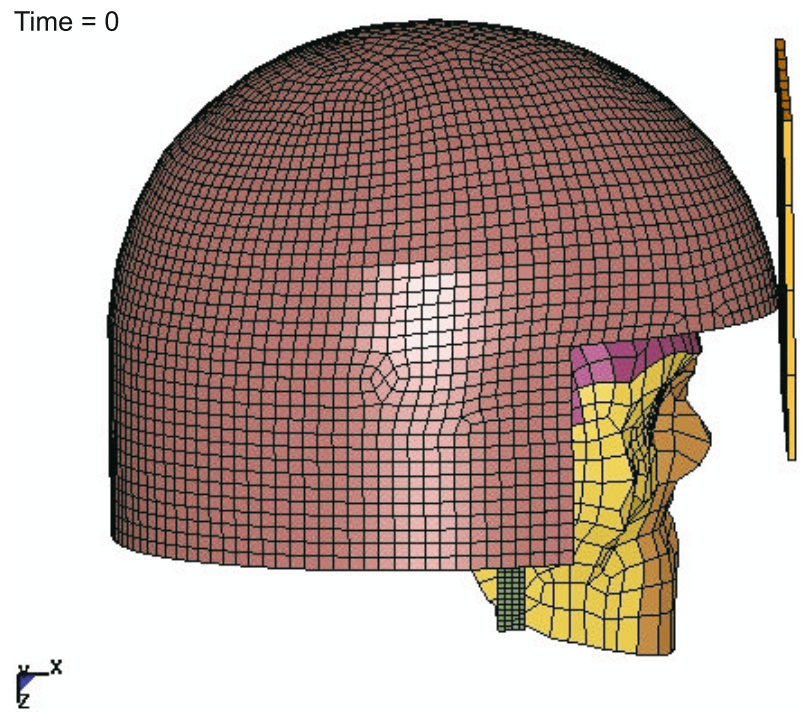

Figure 4. FE model of helmetedhead impact with flat rigid surface at $t=0 \mathrm{sec}$. 


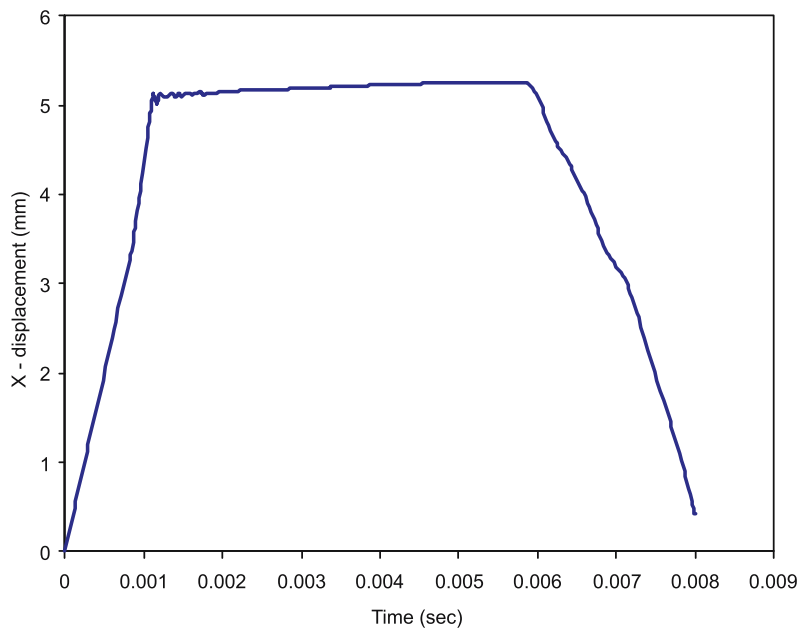

Figure 5. Displacement of the head in the direction of impact without helmet.

In most numerical studies using helmets the head is assumed to be rigid and head injury criterion (HIC) is determined. The purpose of the present paper is to compare the forces and pressures experienced by the head, with and without a helmet, during a frontal impact. For this initial study HIC has not been determined. At present the impact velocities have been kept low at $4 \mathrm{~ms}^{-1}$ to $5 \mathrm{~ms}^{-1}$ because at higher speeds too many oscillations are observed in the contact force for the head without helmet for the mesh size used by us. We propose to increase the velocity in our further studies. The time durations of impact are small and wave propagation rather than structural dynamics is important. As a result, the neck restraint was unlikely to affect the response.

\section{Results and discussion}

The injuries to the head can take various forms such as lacerations of the skin, bone fracture, intracranial injury and brain injury. The forces required to cause a particular injury are variable and very little quantitative information exists about the magnitude of force, stress or strain that will cause a particular injury. However, some experimental measurements on cadavers provide information about forces and pressures for coup and contrecoup injuries to the brain. For a head without helmet (figure 5) the displacement of the head increases very rapidly initially and is almost constant after impact whereas for a helmeted head the displacement rise is much slower. Figures 6,7 and 8 show the impact of helmet-head with the rigid surface at different time steps.

Figures 9 and 10 plot the displacements and velocities of the foam and head for nodes with identical coordinates. When helmet impacts the rigid surface, initially few nodes come in contact with rigid surface and soon acquire a velocity in the opposite direction $(-x$ in figure 10) while still maintaining a contact with the rigid surface. The initial rise and fall of the contact force between helmet and rigid surface shown in figure 11 can be attributed to this. The gap between helmet foam and forehead is $9 \mathrm{~mm}$ to $10 \mathrm{~mm}$. At $t=0.002 \mathrm{~s}$ the head comes in contact with inside of the foam and nodes of the shell again acquire a velocity in the direction of impact $(+x$ in figure 10). This increases the contact area and the contact pressures between the helmet and rigid surface. The contact forces continue to rise till time $0.008 \mathrm{sec}$. 


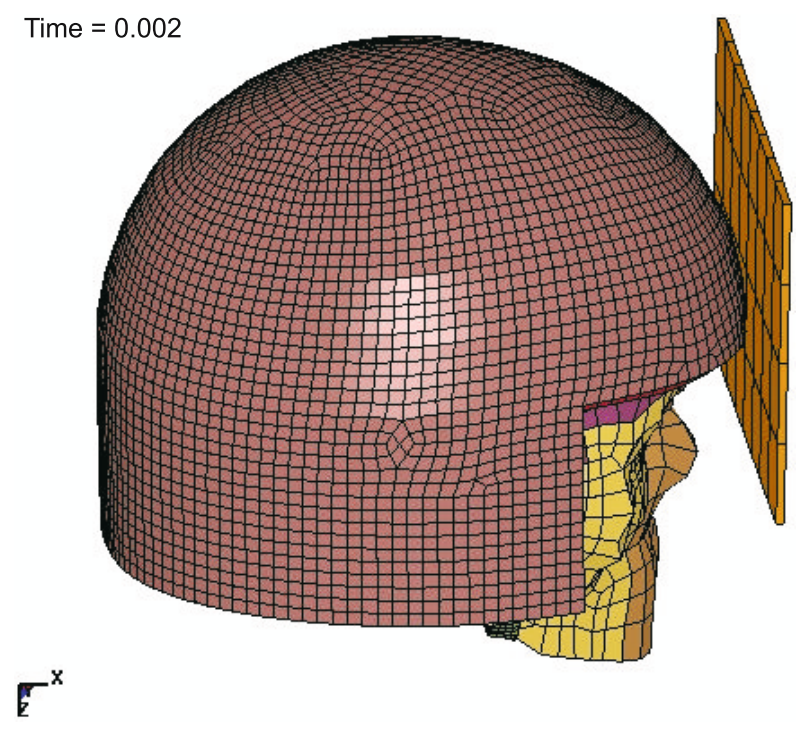

Figure 6. Head is touching the helmet at $t=0.002 \mathrm{sec}$.

Maximum contact forces are reached at $t=0.0062 \mathrm{~s}$ and then the helmet starts rebounding. At this time, the foam and head start separating and the force drops. The separation between the two surfaces is also evident in figure 10 from the direction of the velocities of foam and head nodes with identical positions. Beyond 0.008 seconds the foam liner recovers with a constant velocity of $0.25 \mathrm{~ms}^{-1}$ whereas the rebound velocity of the head is higher leading to separation of surface and loss of contact.

Figure 12 shows the contact force between the head and the rigid surface (for a head without helmet) and between the helmet liner and the head. At an impact velocity of $4 \mathrm{~ms}^{-1}$ the maximum contact force on the head without helmet is about $7800 \mathrm{~N}$ as compared to $3500 \mathrm{~N}$

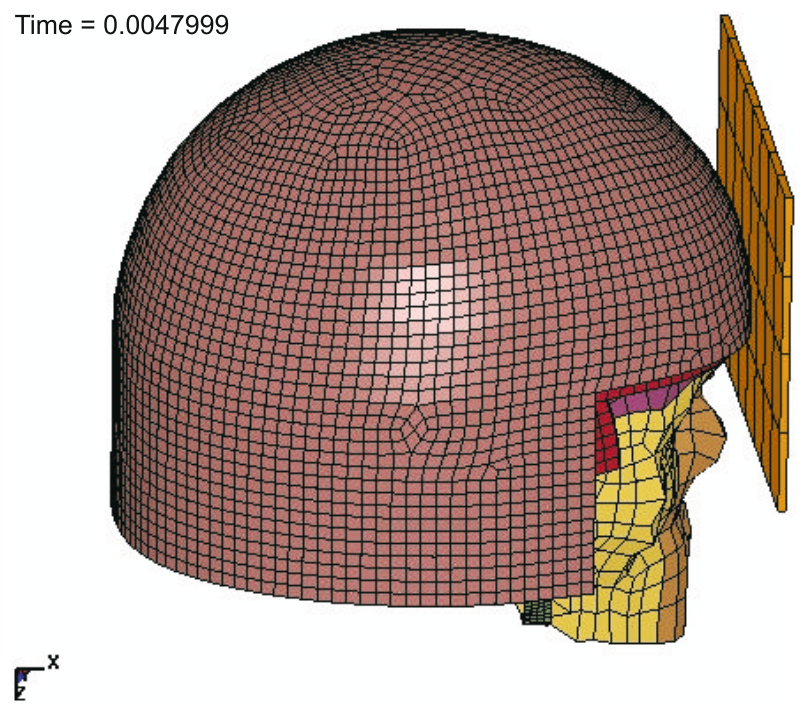

Figure 7. Maximum contact forces are rising at $t=0.0048 \mathrm{sec}$. 




$5^{x}$


Figure 8. Helmet is lifting up from the back side while rebounding.
Figure 9. Displacement of head and foam in the direction of helmet impact.

Figure 10. Velocity of head and foam during the impact. 


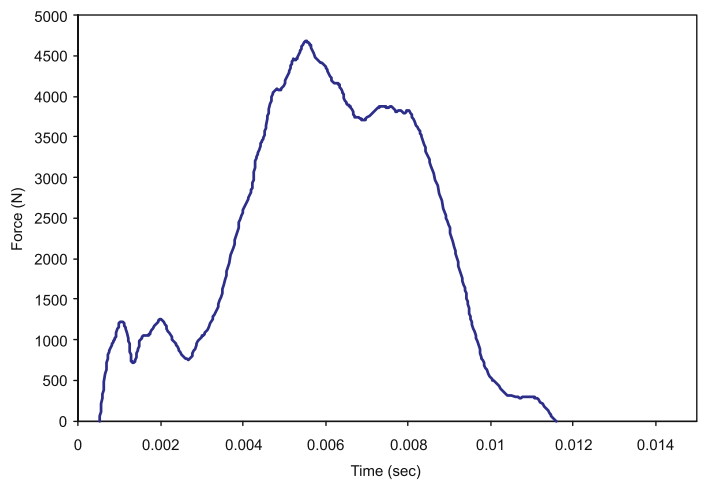

Figure 11. Contact force between rigid surface and helmet shell at velocity $4 \mathrm{~ms}^{-1}$.

to $5400 \mathrm{~N}$ (depending on density of foam) for a helmeted head. The maximum contact forces between the helmet shell and a rigid surface is approximately $5000 \mathrm{~N}$ about $2800 \mathrm{~N}$ lower than that experienced by a bare head although the shell material has a much higher modulus compared to the skull. These lower forces are probably due to the curvature of the shell and presence of foam which reduces the overall stiffness of the shell.

Figure 12 also compares contact forces between helmets with foams of different densities. The higher the density of foam, the higher the contact forces on the head and it seems that lower density foam works better than higher density foam. However, at higher velocities the lower density foam may crush completely and touch the helmet shell resulting in a higher contact force on the head. The contact forces did not change significantly as the thickness of the foam was increased. The thickness of foam is important only when velocities are high and bottoming out of the foam occurs. Since the impact velocities were low bottoming out was not observed and the contact forces are almost independent of thickness. Figure 13 shows the contact forces for impact velocity of $5 \mathrm{~ms}^{-1}$. The maximum contact force on the head without helmet increases from $7800 \mathrm{~N}$ to $10000 \mathrm{~N}$ and for a head with helmet the force changed from $5400 \mathrm{~N}$ to $6200 \mathrm{~N}$. The duration of contact between the rigid surface and the helmet is about $7 \mathrm{~ms}$, while the duration of contact between the helmet and head is about $4 \mathrm{~ms}$.

Figure 14 shows the displacement of a node on the front side of the CSF and the brain. Initially both are moving together till time $t=0.003 \mathrm{sec}$ but later the CSF starts compressing

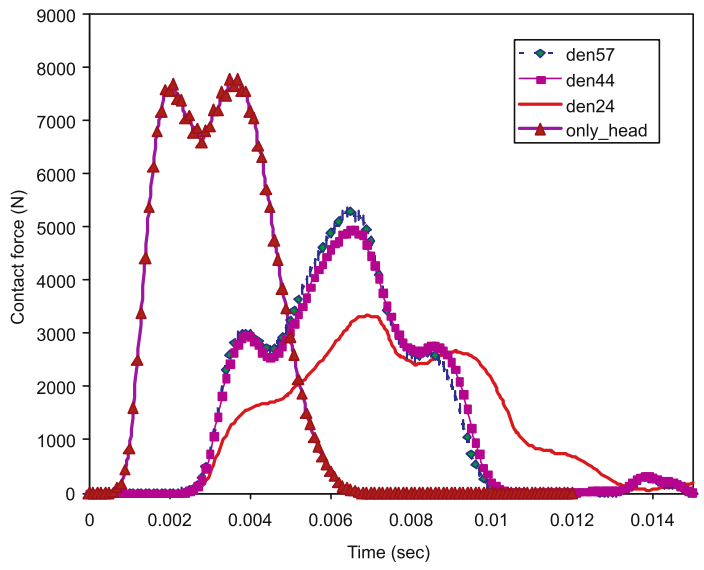

Figure 12. Contact force between rigid surface-head and head-foam at impact velocity $4 \mathrm{~ms}^{-1}$. 

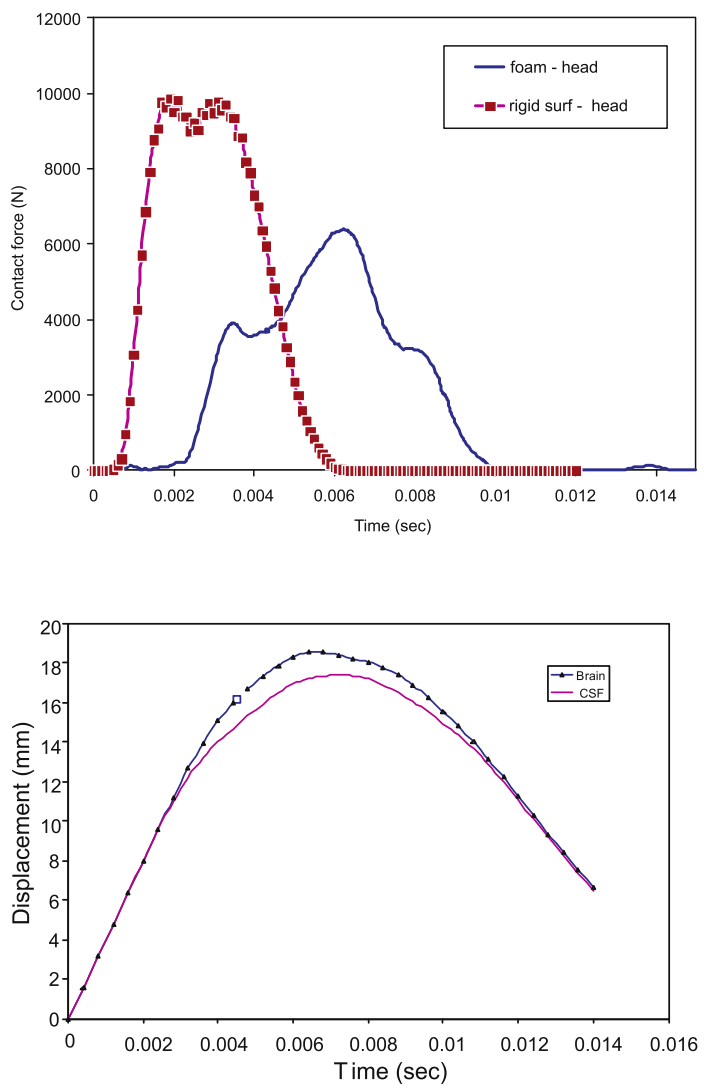

Figure 13. Contact force between rigid surface-head and head-foam at impact velocity $5 \mathrm{~ms}^{-1}$.
Figure 14. Displacements of brain and CSF in the direction of impact at velocity $4 \mathrm{~ms}^{-1}$.

and the brain still moves in the impact direction. The displacement in the brain is more but because of the lower modulus of the brain, and because the stresses are low and as are the pressures. Importantly, the contact area between the foam and head is approximately $5000 \mathrm{~mm}^{2}$ and is much larger as compared to the contact area between head and rigid surface without helmet, which is $2100 \mathrm{~mm}^{2}$, so that contact pressures on the surface of the head and intracranial pressures, as shown in figures 15 and 16, for a helmeted head are much lower.

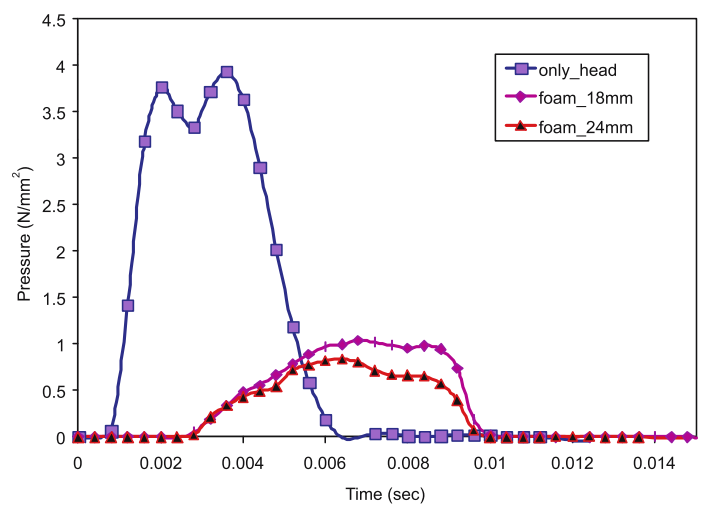

Figure 15. Hydrostatic pressure on the surface of head with and without helmet. 

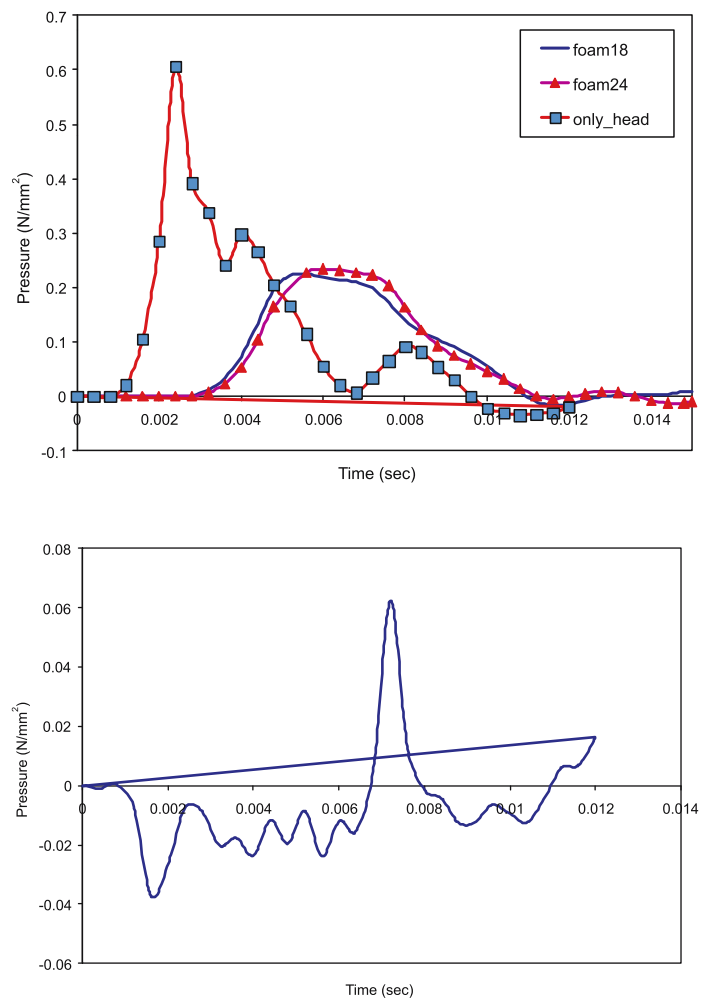

Figure 16. Intracranial pressure on the coup side without and with helmet of different foam thicknesses.

Figure 17. Pressure on Contrecoup side of the head without helmet.

The maximum contact pressures on the coup side are almost five times lower and intracranial pressures are three times lower when the helmet is present.

The maximum contact pressures in the region of impact decreases from $1 \mathrm{MPa}$ to $0.8 \mathrm{MPa}$ as the thickness of the foam is increased from 18 to $24 \mathrm{~mm}$. The contrecoup pressures, compared in figure 17, are similar whether the helmet is there or not. The bare head impact pressure at contrecoup site is $-0.038 \mathrm{MPa}$ and when it impacts with helmet it is around $-0.015 \mathrm{MPa}$ (figures 17 and 18). And the trend of contrecoup pressures is similar with change in thickness of liner and also with the change of liner density.

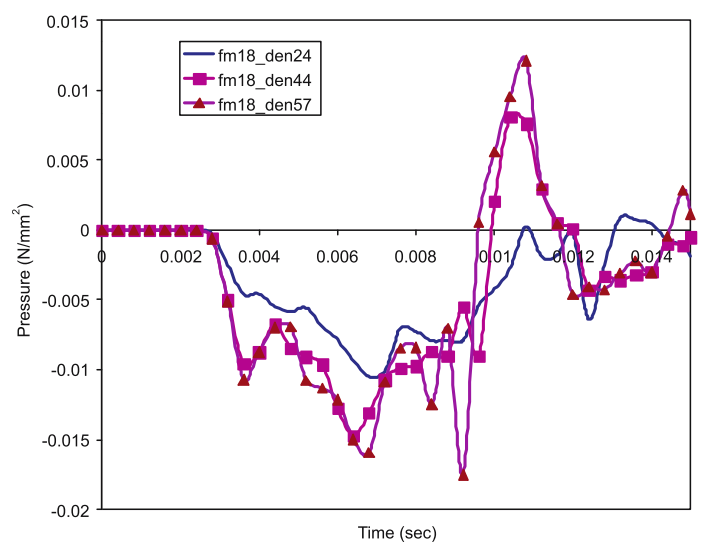

Figure 18. Pressure on Contrecoup side of the head with helmet. 


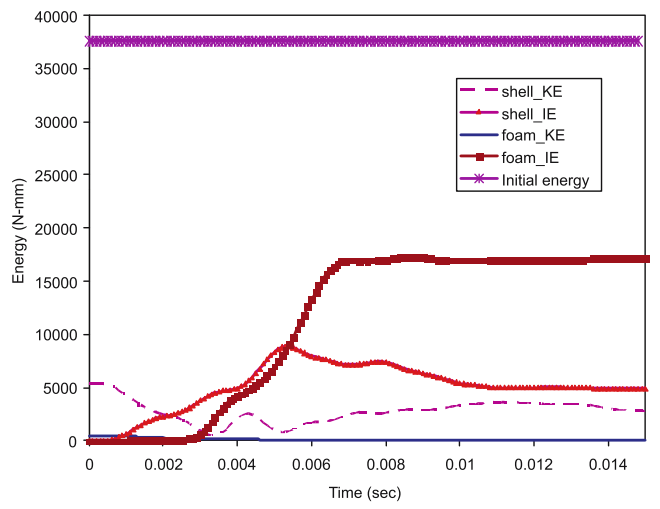

Figure 19. Initial energy and energy variation with time in the helmet outer shell and foam.

Figure 19 plots the internal energy absorbed by the foam and shell and the kinetic energy of the head versus time during the impact. The initial kinetic energy of the head of mass $4.0 \mathrm{~kg}$ is approximately $37 \mathrm{KJ}$. The foam of density $44 \mathrm{~kg} \mathrm{~m}^{-3}$ absorbs almost $17 \mathrm{KJ}$ of the energy whereas the shell absorbs $9 \mathrm{KJ}$ of the energy. The shell being elastic returns part of the energy on rebound.

\section{Conclusions}

A comparison is made between the behavior of the human head with and without a helmet during frontal impact. Unlike previous studies where the head was assumed to be rigid, here the head is assumed to be deformable and various parts of the head are modelled using the finite element method. The velocities were kept low at $4 \mathrm{~ms}^{-1}$ to $5 \mathrm{~ms}^{-1}$ so that the force-time curve of the head without helmet does not show oscillations. The contact forces and pressures for the head without helmet were compared with values in the literature and showed a good match. The helmet absorbs $80 \%$ of the kinetic energy of the system with the foam absorbing almost $67 \%$ and the shell about $33 \%$ of this absorbed energy. The coup pressures in the head with helmet are substantially reduced although the contrecoup pressures did not change much when compared without a helmet.

For the velocities studied the thickness of foam did not have a significant effect on the contact forces. The lowest density foam gave the lowest contact forces and coup pressures. However, at higher velocities there is a possibility of the foam bottoming out leading to higher forces. Previous models using rigid head forms used HIC or deceleration as a measure for injury and brought out the necessity of using a helmet during an accident. The present FE modelling, which treats the head as deformable, shows a substantial reduction in intracranial pressures due to impact by use of helmets. The present study restricted itself to front impacts when the helmet is tied properly using a strap very little relative movement between the head and helmet occurs. Side impact and oblique impact are the next obvious extensions of this work. Preliminary investigations indicate that in oblique impact angular accelerations will have an effect on coup pressures.

\section{References}

Becker B Edward 1998 Helmet development and standards. Biomedical and Health Research, Frontiers in Head and Neck Trauma, IOS press, 21: 113-130 
Bowman M Bruce, Lawrence W Schneider, Paul R Rohr and Dinesh Mohan 1982 Simulation of head/neck impact responses for helmeted and unhelmeted motorcyclists, SAE Inc., Paper No. 811029, 3318-3343

Brands D W A, Thunnissen, Wismans J S H M 1996 Modelling head injury countermeasures: a 3D helmet model. AGARD specialists meeting on impact head injury, Mescalero, New Mexico, TNO crash safety research centre

Hamouda A M S et al 2007 A new motorcycle helmet liner material: The finite element simulation and design of experiment optimization. Materials \& Design 28(1): 182-195

Horgan T J, Gilchrist M D 2003 The creation of three-dimensional finite element models for simulating head impact biomechanics. Int. J. Crashworthiness 8(4): 354

Huston R L, Sears J 1981 Effect of protective Helmet mass on Head/Neck dynamics. J. Biomech. Engg. Trans. 103: 18-23

Mohan D, Kothiyal K P 1984 Helmet and head injury study of crash involved motorcyclists in Delhi, IRCOBI conf. Proc. 65-77

Remy Willinger, Baye M Diaw, Ho-Sung Kang 2000 Finite element modelling of skull fractures caused by direct impact. Int. J. Crashworthiness 5: 3

Ruan J S, Khalil T, King AI 1994 Dynamic response of the human head to impact by three-dimensional finite element analysis. J. Biomech. 116(1): 44-50

Yettram A L, Godfrey N P M, Chinn B P 1994 Materials for motorcycle crash helmets - a finite element parametric study. Plastics, Rubber and Composites Processing and Applications 22(4): 215-221

Zong Z, Lee H P, Lu C, 2006 A three-dimensional human head finite element model and power flow in a human head subjected to impact loading. J. Biomech. 39(2): 284-292 